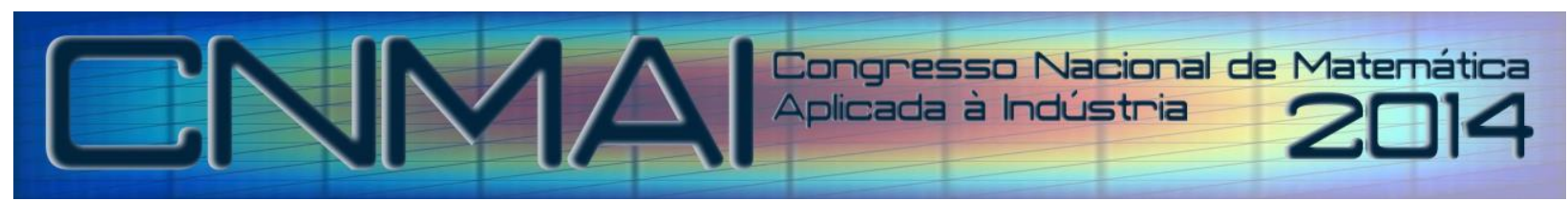

18 a 21 de novembro de 2014, Caldas Novas - Goiás

\title{
ESTIMAÇÃO DE PARÂMETROS DE UM MÓDULO TERMOELÉTRICO COM ALGORITMO DAS ABELHAS PSO
}

\author{
Daniel Ricardo Ojeda Giratá, daniel.ojeda@ufabc.edu.br ${ }^{1}$ \\ Omar Alexander Chura Vilcanqui, omarchu@gmail.com ${ }^{2}$ \\ Luiz Alberto Luz de Almeida, luiz.almeida@ufabc.edu.br ${ }^{3}$ \\ ${ }^{1}$ Universidade Federal do ABC - Pós-Graduação em Engenharia Elétrica ... http://www.ufabc.edu.br/
${ }_{2}^{2}$ Universidade Federal da Bahía - Pós-Graduação em Engenharia Industrial ... http://www.ufba.br/
${ }^{3}$ Universidade Federal do ABC - Pós-Graduação em Engenharia Elétrica ... http://www.ufabc.edu.br/
}

Resumo: Módulos termoelétricos (TEM Thermoelectric Modules) são utilizados na geração de energia elétrica e na construção de câmaras térmicas para caracterização de materiais como ligas de memória de forma (SMA- Smart Memory Allow), dentre outros. Para ter uma correta representação dos TEM é necessária a identificação de um modelo que consiga representar o seu funcionamento, tanto em corrente contínua como em demais frequências relevantes. No presente trabalho, é proposto um modelo para a representação do TEM considerando as não linearidades deste. Métodos clássicos de estimação para modelos lineares nos parâmetros não se aplicam para o modelo proposto. Para obtenção dos valores dos parâmetros do TEM, este é excitado com ruído branco e a resposta é utilizada para o algoritmo de PSO (Particle swarm optimization) fazer a estimação. O modelo escolhido para a caracterização do TEM é não-linear com oito parâmetros, que representam elementos do TEM tais como: a camada superior, a placa superior, camada central, placa inferior e o dissipador de calor do TEM, sendo os oito parâmetros calculados pelo PSO. O ruído branco de excitação é antes filtrado, resultando em um sinal dinamicamente persistente com componentes de altas e baixas frequências, de tal forma que o TEM seja bem caracterizado. Resultados de simulações mostram a efetividade do PSO no cálculo de parâmetros do modelo TEM.

Palavras-chave: módulo termoelétrico, estimação de parâmetros, identificação de sistemas não-lineares, otimização por enxame de abelhas (PSO), metaheurística.

\section{INTRODUÇÃO}

Nos últimos anos, tem havido um interesse crescente na área da identificação e modelagem dos sistemas, embora os sistemas lineares foi incialmente o foco dos pesquisadores mas recentemente, tem surgido maior ênfase sobre os sistemas com características dinâmicas. Os modelos obtidos a partir da identificação e modelagem dos sistemas são usados posteriormente em atividades de controle, confiabilidade e predição de falas entre outras, No presente trabalho descreve-se a modelagem e estimação dos parâmetros de um módulo termoelétrico (TEM) considerando as características não-lineares que apresenta este dispositivo, sendo depois implementado em aplicações para caracterização de materiais e identificação de geradores termoelétricos.

Um módulo termoeléctrico é um componente eletrônico construído a partir de semicondutores, este dispositivo permite a manipulação da temperatura em ambos os lados, um lado quente e o outro frio, pelas suas características, sua fácil adaptação e estrutura sem peças móveis que o torna um dispositivo de estado sólido, o uso dos módulos termoelétricos em diversos setores da indústria está em constante ascensão, principalmente como elemento de refrigeração, mas também são usados como mecanismo para a geração de energia partindo de gradientes de temperatura. Aplicações na área de controle de temperatura (Almeida, 2003) tais como as câmaras térmicas onde o calor expulso é implementado para a caracterização de materiais, quando expostos a altas temperaturas, suas alterações devem ser de forma precisa e controlada, consequentemente, é necessário conhecer os parâmetros intrínsecos nãolineares em conjunto com as características dinâmicas do TEM em condições específicas. 
Algumas das técnicas utilizadas para obtenção dos parâmetros térmicos dinâmicos dos TEM são baseados em medições experimentais de tensão, corrente e temperatura de um TEM em funcionamento (Mitrani et al., 2005), em procedimentos analíticos baseados nas curvas de performance fornecidas pelos fabricantes (Palacios et al., 2009), nos resultados do algoritmo de otimização TLBO, para estabelecer os parâmetros ideais na construção de um arranjo de dois TEM quando conectados eletricamente em série (Venkata e Vivek, 2012), ou na implementação do algoritmo genético GA (Almeida, 2003).

O método de otimização por enxame de partículas ou PSO (Particle Swarm Optimization) desenvolvido no início dos anos 90 (Kennedy e Eberhart, 1955) tem sido amplamente implementado em vários casos de otimização como descrito em (Poli, 2007). PSO é uma técnica de otimização estocástica robusta, não-linear com base no comportamento dos enxames de abelhas em procura de pólen, com este método é possível obter soluções de ótima qualidade, em intervalos de tempos curtos e características de convergência estáveis (Mondal et al., 2012).

Neste trabalho, descreve-se a metodologia da estimação dos oito parâmetros dinâmicos do TEM mostrado na Fig. 1 utilizando a técnica do algoritmo PSO. Um arranjo de dois TEM quando conectados em cascata térmica será usado, junto com um controlador de corrente para o sinal de excitação que é embarcado no microcontrolador de 32 bits Arduino Due. O sinal de excitação tipo ruído branco gerado pelo computador é transferido ao microcontrolador para fornecer a potência necessária na excitação do TEM mediante um amplificador baseado em um inversor DC-AC. A validação do método, é realizada com os resultados obtidos na estimação quando comparados com os parâmetros previamente estabelecidos do modelo assumido como real.
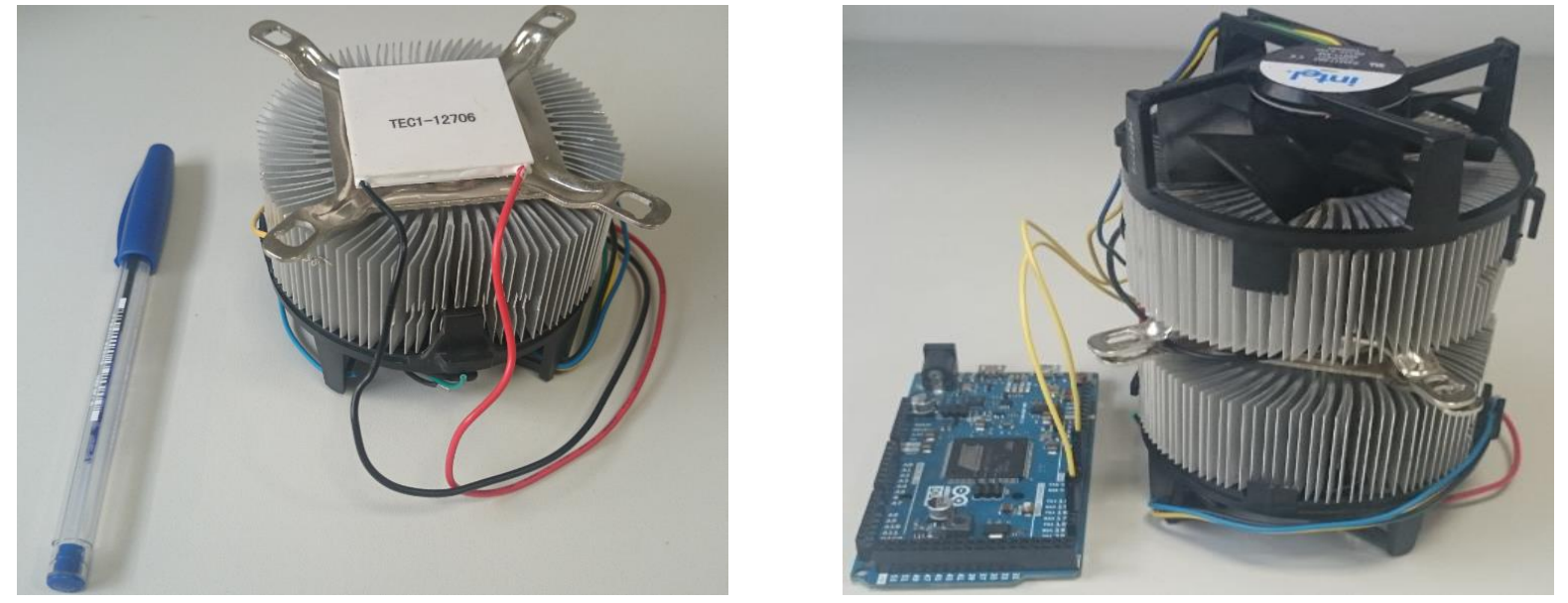

Figura 1. Módulo termoelétrico de fabricação da HB Coorporation modelo TEC1-12706.

\section{MÓDULO TERMOELÉTRICO}

O dispositivo TEM consiste em vários semicondutores conectados eletricamente em série e prensados entre duas placas de cerâmica como mostrado na Fig. 2. Quando conectados a uma fonte corrente de alimentação contínua, a corrente passa pelos elementos produzindo o bombeamento de calor de um lado para outro. Como consequência, isto cria um lado quente e um lado frio no TEM (Almeida, 2003).

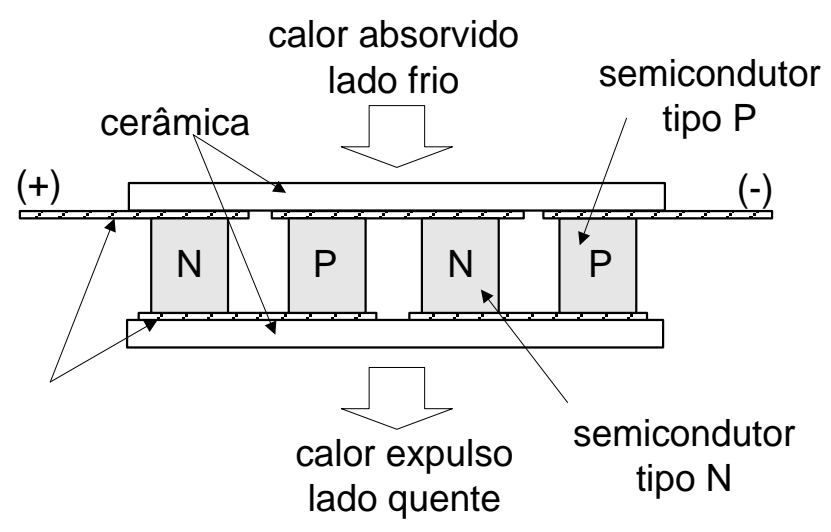

Figura 2. Representação gráfica de um Módulo Termoelétrico (Almeida, 2003).

Em (Almeida, 2003) é proposto um modelo dinâmico não-linear para um módulo termoelétrico, em conjunto com uma câmara térmica. Para condições específicas, é obtido um modelo simplificado para o TEM, e os parâmetros obtidos a partir de uma corrente de excitação Ic e do comportamento da temperatura Tx. 


\section{MODELO DINÁMICO DA CÂMARA TÉRMICA}

O comportamento térmico de um dispositivo pode ser estudado através da equação de balanço de calor (Meijer et al., 1994 e Dalola et al., 2009). A quantidade de calor Q em [J] armazenada em um corpo pode ser calculada através da Eq. (1):

$$
\frac{d Q}{d t}=(P e-P s)+(P g-P a)
$$

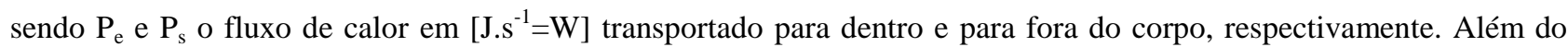
fluxo que passa através do corpo, os fluxos de calor $\mathrm{P}_{\mathrm{g}}$ e $\mathrm{P}_{\mathrm{a}}$ podem ser gerados ou absorvidos respectivamente dentro deste corpo. Quando há fluxo de calor em um corpo, há alteração na temperatura do mesmo (Almeida, 2003). Para expressar esta mudança na temperatura do corpo, a Eq. (1) pode ser escrita na forma da Eq. (2):

$$
C \frac{d T}{d t}=(P e-P s)+(P g-P a)
$$

sendo $\mathrm{C}=\mathrm{m} . \mathrm{c}$ a capacitância térmica do corpo em $\left[\mathrm{J} \cdot \mathrm{K}^{-1}\right], \mathrm{m}$ a massa do corpo em $[\mathrm{kg}]$ e c seu calor específico em $\left[\mathrm{J}_{\mathrm{kg}} \mathrm{k}^{-1} \cdot \mathrm{K}^{-1}\right]$. O fluxo de calor transportado para dentro e para fora de um corpo se da através de três formas distintas: condução; convecção e radiação (Almeida, 2003). Um fluxo P entre dois corpos produz uma diferença de temperatura $\Delta T$, que pode ser representada pela condutância térmica $\mathrm{G}$ entre eles definida, como mostrado na Eq. (3):

$$
G=\frac{P}{\Delta T}\left[W \cdot K^{-1}\right]
$$

As características para a construção de uma câmara térmica e as respectivas grandezas associadas a esta são apresentadas na Fig. 3. Sendo Tx e Cx a temperatura e a capacitância térmica da placa superior do TEM, Ty e Cy a temperatura e a capacitância térmica da placa inferir do TEM, Gyd a condutância térmica entre a placa inferior do TEM e o dissipador de calor, Cd a capacitância térmica do dissipador de calor, Rxy e Gxy a resistência e condutância térmica entre as placas superior e inferior do TEM, Gda a condutância térmica entre o dissipador de calor e o meio ambiente, Gzd a condutância térmica entre a resistência de aquecimento e o dissipador de calor, Gsx a condutância térmica entre o substrato e a placa superior do TEM e Gm e Cm a condutância e capacitância térmica do termistor. Como primeira abordagem, a análise apresentada no presente trabalho foi desenvolvida considerando um TEM, as variações na estimação dos parâmetros geradas pela influência do TEM auxiliar fazem parte das seguintes investigações.

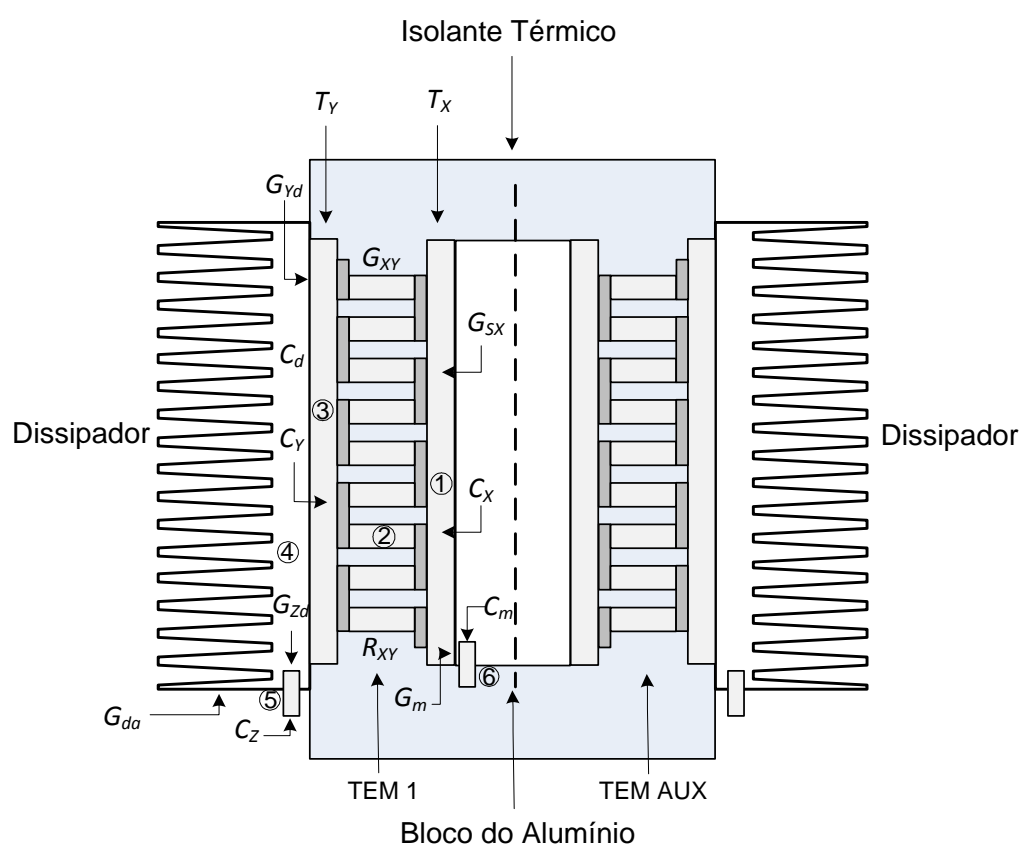

Figura 3 - Descrição das grandezas térmicas da planta. 1) Placa superior do TEM; 2) Camada central do TEM; 3) Placa inferior do TEM; 4) Dissipador de calor; 5) Resistor de aquecimento do dissipador de calor; 6) Termistor para medição de temperatura. 
Quando uma corrente $\mathrm{I}_{\mathrm{C}}$ passa pelo TEM, o fluxo de calor produzido nas placas superior e inferior do TEM são respectivamente representadas pelas equações Eq. (4) e Eq.(5) (Rowe, 1995, Huang e Duang, 2000 e Chávez et al., 2000):

$$
\begin{aligned}
& \frac{d Q_{X}}{d t}=P_{X}-G_{X Y}\left(T_{X}-T_{Y}\right)\left[J \cdot S^{-1}\right] \\
& \frac{d Q_{Y}}{d t}=P_{Y}+G_{X Y}\left(T_{X}-T_{Y}\right)\left[J \cdot S^{-1}\right]
\end{aligned}
$$

sendo $G_{x y}\left(T_{x}-T_{y}\right)$ o fluxo de calor por condução entre as placas superior e inferior do TEM e $P_{x}$ e $P_{y}$ são as potências ativas de calor, dadas pelas equações Eq.(6) e Eq.(7) respectivamente:

$$
\begin{aligned}
& P_{X}=I_{C}{ }^{2} \frac{R_{X Y}}{2}+K_{S} T_{X} I_{C}[W] \\
& P_{Y}=I_{C}{ }^{2} \frac{R_{X Y}}{2}-K_{S} T_{Y} I_{C}[W]
\end{aligned}
$$

para as quais $R_{x y}$ é a resistência elétrica entre as placas superior e inferior do TEM em [Ohm] e $k_{s}$ é o coeficiente de Seebeck em $\left[\mathrm{V} . \mathrm{K}^{-1}\right.$ ]. O termo $\mathrm{I}_{\mathrm{c}}{ }^{2} \mathrm{R}_{\mathrm{xy}}$ está relacionado com o aquecimento do TEM por efeito Joule. Metade desta potência Joule $\mathrm{I}_{\mathrm{c}}{ }^{2} \mathrm{R}_{\mathrm{xy}} / 2$ é atribuída a cada uma das placas. Os termos $+\mathrm{k}_{\mathrm{s}} \mathrm{T}_{\mathrm{x}} \mathrm{I}_{\mathrm{c}}$ e $-\mathrm{k}_{\mathrm{s}} \mathrm{T}_{\mathrm{y}} \mathrm{I}_{\mathrm{c}}$ estão relacionados com o efeito Peltier. Se a corrente flui numa determinada direção tal que $I_{c}>0$, a placa superior gera o fluxo de calor $k_{s} T_{x}\left|I_{c}\right|$ em adição à potência Joule $\mathrm{I}_{\mathrm{c}}{ }^{2} \mathrm{R}_{\mathrm{xy}} / 2$ e causa um aumento na temperatura $T_{\mathrm{x}}$. Simultaneamente mantendo $\mathrm{I}_{\mathrm{c}}>0$, a placa inferior absorve um fluxo de calor $-\mathrm{k}_{\mathrm{s}} \mathrm{T}_{\mathrm{x}}\left|\mathrm{I}_{\mathrm{c}}\right|$, somado a potência Joule $\mathrm{I}_{\mathrm{c}}{ }^{2} \mathrm{R}_{\mathrm{xy}} / 2$.

A placa inferior do TEM foi colocada em contato térmico com um dissipador de calor de massa muito maior do que a massa do TEM. Dessa forma o fluxo de calor absorvido ou gerado na placa inferior escoa para o dissipador, mantendo assim a temperatura $T_{y}$ num valor aproximadamente constante, igual a temperatura do dissipador $T_{d}$ (Almeida, 2003). Como a temperatura $\mathrm{T}_{\mathrm{y}}$ é mantida constante a temperatura $\mathrm{T}_{\mathrm{x}}$ sofre um aumento quando $\mathrm{I}_{\mathrm{c}}>0$. Este é o efeito de aquecimento da placa superior do TEM. Caso o sentido da corrente seja invertido $\left(\mathrm{I}_{\mathrm{c}}<0\right)$ a placa superior do TEM passa a absorver um fluxo de calor $-k_{s} T_{x}\left|I_{c}\right|$, somado à potência Joule $I_{c}{ }^{2} R_{x y} / 2$ o que causa uma redução na temperatura $T_{x}$ em relação a $T_{d}$. Na Fig. 4. é mostrado o comportamento da potência ativa de calor $P_{x}$ em função da corrente $I_{C}$ no TEM.

Nas regiões 1 e 3 o fluxo de calor $P_{x}$ gerado na placa superior é positivo, o que causa um aumento na temperatura $\mathrm{T}_{\mathrm{x}}$. Nota-se que na região 3 mesmo para corrente $\mathrm{I}_{\mathrm{c}}<0$ o fluxo de calor $\mathrm{P}_{\mathrm{x}}$ pode se tornar positivo, produzindo assim um aumento em $T_{x}$. Na região 2, o fluxo de calor Px é negativo o que causa uma redução na temperatura $T_{x}$.

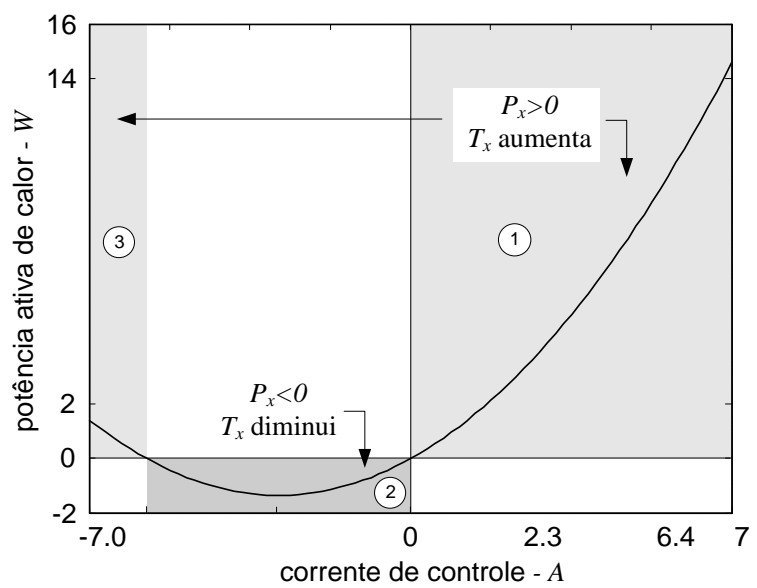

Figura 4. Potência ativa de calor Px em função da corrente de controle Ic no TEM (Almeida, 2003).

Em (Almeida, 2003) propõe-se o seguinte modelo de parâmetros concentrados na câmara térmica conforme descrito na Eq. (8):

$$
\begin{aligned}
& C_{X} \dot{\mathrm{T}}_{X}=P_{X}+G_{S X}\left(T_{S}-T_{X}\right)-G_{X Y}\left(T_{X}-T_{Y}\right) \\
& C_{Y} \dot{\mathrm{T}}_{Y}=P_{Y}+G_{X Y}\left(T_{X}-T_{Y}\right)-G_{Y d}\left(T_{Y}-T_{d}\right) \\
& C_{d} \dot{\mathrm{T}}_{d}=P_{Z}+G_{Y d}\left(T_{Y}-T_{d}\right)-G_{d a}\left(T_{d}-T_{a}\right)
\end{aligned}
$$

O comportamento da temperatura $\mathrm{T}_{\mathrm{x}}$ a ser controlada pelo efeito da corrente de excitação $\mathrm{I}_{\mathrm{C}}$, pode ser representado pelas equações apresentadas na Eq. (9) colocadas na forma: 


$$
\begin{gathered}
\dot{\mathrm{T}}_{X}=\frac{1}{C_{X}}\left(I_{C}{ }^{2} \frac{R_{X Y}}{2}+K_{S} T_{X} I_{C}\right)-\frac{G_{X Y}}{C_{X}} T_{X}+\frac{G_{X Y}}{C_{X}} T_{Y} \\
\dot{\mathrm{T}}_{Y}=\frac{1}{C_{Y}}\left(I_{C}{ }^{2} \frac{R_{X Y}}{2}-K_{S} T_{Y} I_{C}\right)+\frac{G_{X Y}}{C_{Y}} T_{X}-\frac{\left(G_{X Y}+G_{Y d}\right)}{C_{Y}} T_{Y}+\frac{G_{Y d}}{C_{Y}} T_{d} \\
\dot{\mathrm{T}}_{d}=\frac{P_{Z}}{C_{d}}+\frac{G_{Y d}}{C_{d}} T_{Y}-\frac{\left(G_{X d}+G_{d a}\right)}{C_{d}} T_{d}+\frac{G_{d a}}{C_{d}} T_{a}
\end{gathered}
$$

Conforme descrito anteriormente, existe uma dinâmica não-linear que relaciona a corrente e a temperatura, o que dificulta a estimação dos parâmetros do TEM pelos métodos exatos, porém propõe-se a implementação do algoritmo PSO dadas suas características no desenvolvimento de problemas de otimização de alta complexidade (Poli, 2007).

\section{OTIMIZAÇÃO BASEADA NA METAHEURÍSTICA DAS ABELHAS}

O método de otimização por enxame de partículas ou PSO foi originalmente desenvolvido pelo psicólogo-sociólogo Jammes Kennedy e pelo engenheiro eletrônico Russell Eberhart no ano 1995, é uma técnica metaheurística baseada nas populações e inspirada no comportamento social do voo das abelhas em procura do alimento ou o movimento dos cardumes de peixes. No método PSO cada partícula faz parte de uma possível solução representada por sua posição em um espaço de busca determinado, a implementação do algoritmo pode se descrever como apresentado na Fig. 5 (Kennedy e Eberhart, 1955).

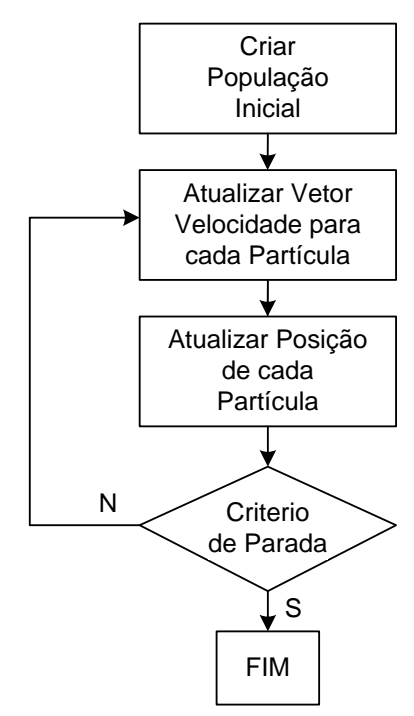

Figura 5. Fluxograma do algoritmo PSO

O primeiro passo é gerar as $\mathrm{N}$ partículas que formarão o "enxame" com suas respectivas posições. Iniciando os valores dos parâmetros a serem otimizados, atribuindo valores aleatórios nos vetores de posição e velocidade ou também, arbitrar velocidades iniciais para cada partícula. O algoritmo manter-se-á ativo, calculando a função objetivo que determina as discrepâncias entre os valores do modelo real e o modelo experimental, atualizando os vetores de velocidade e posição ciclicamente até que seja atingido a condição de convergência e de parada (número máximo de iterações atingido ou partícula com aptidão desejada, etc.) (Vasconcelos, 2007).

\section{ESTIMAÇÃO DOS PARÂMETROS DO MÓDULO TERMOELÉTRICO}

O método implementado na estimação dos parâmetros do TEM, baseia-se no modelo Entrada-Saída, onde a relação entre um sinal de excitação e a influência gerada na resposta dinâmica em um sistema determinado é analisada. Uma das exigências fundamentais é a implementação de um sinal dinamicamente persistente, a efetividade do método depende em grande parte do sinal escolhido. Este deve conter o número suficiente de harmônicos, necessários para excitar todos os modos dinâmicos do TEM e assim caracterizar e identificar o modelo (Ljung, 1987). Na Fig. 6 é apresentado o sinal de corrente de excitação aplicado no TEM que é utilizado no presente trabalho, este é um ruído branco que foi previamente filtrado, resultando em um sinal com componentes de altas e baixas frequências para que o TEM seja bem caracterizado. O sinal filtrado terá um espectro com banda limitada e assim evitar os problemas numéricos relativos ao passo de integração, a função de transferência do filtro utilizado é apresentada na Eq. (10) sendo $\omega_{0}=2 \pi$.f e $\mathrm{f}=0,1 \mathrm{~Hz}$ 


$$
H(s)=\left(\frac{\omega_{0}}{s+\omega_{0}}\right)^{4}
$$

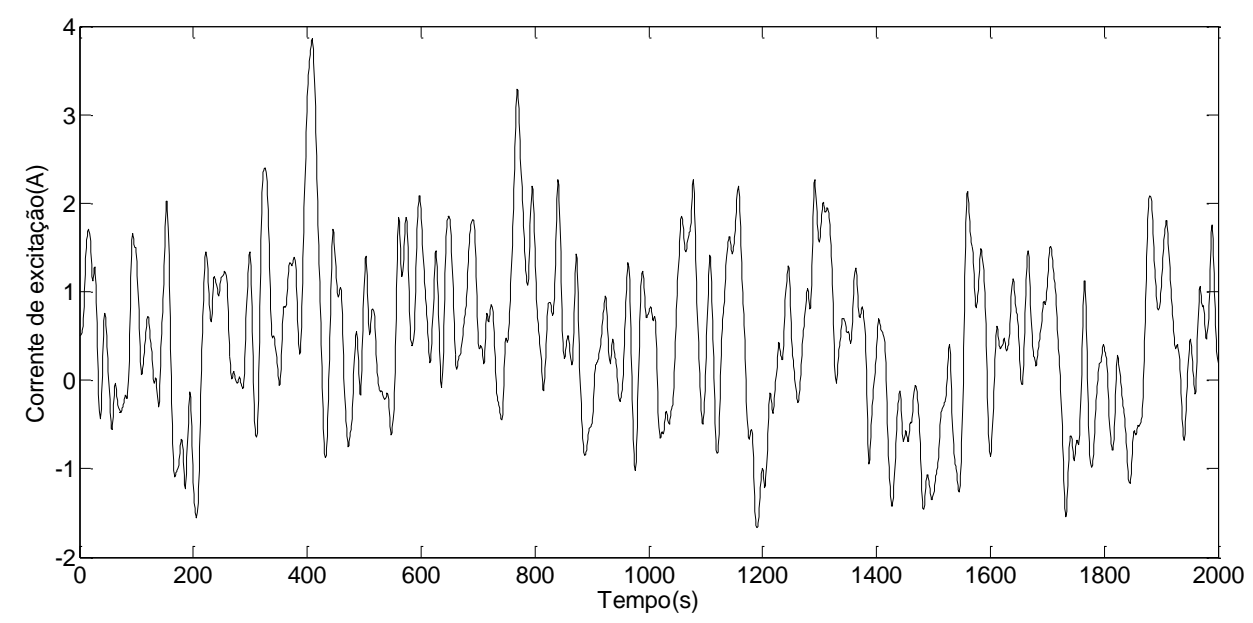

Figura 6. Sinal de excitação utilizado, Corrente $\mathbf{I}_{\mathrm{C}}$.

No diagrama de blocos apresentado na Fig. 7, mostra-se o esquema de implementação do algoritmo PSO para estimação dos parâmetros térmicos dinâmicos do modelo não linear do TEM estabelecidos na Eq. (9), onde um mesmo sinal na entrada do sistema alimenta o modelo real e o modelo estimado obtendo-se os valores iniciais dos vetores posição e velocidade em cada uma das partículas que fazem parte do enxame. Os sinais resultantes na saída do modelo real e o modelo estimado são relacionados quando comparados com a função objetivo, o resultado da operação fornecera o módulo PSO até ser atingido o critério de parada.

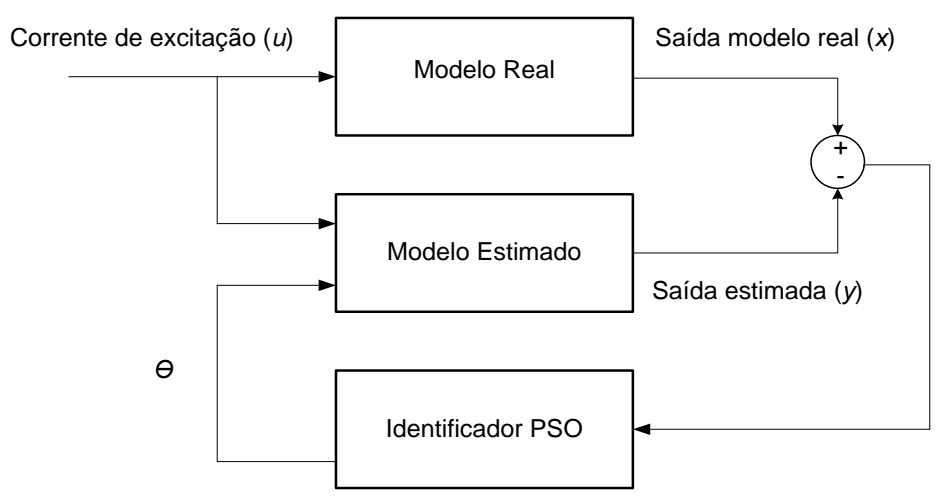

Figura 7. Método de estimação dos parâmetros do TEM baseado no algoritmo PSO (Therdbankerd et al., 2010).

Foram consideradas as restrições apresentadas na Eq. (11) para que os parâmetros calculados sejam fisicamente possíveis.

$$
\begin{aligned}
& K_{S}{ }^{\min }<K_{S}<K_{S}{ }^{\max } \\
& R_{X Y}{ }^{\text {min }}<R_{X Y}<R_{X Y}{ }^{\text {max }} \\
& C_{d} \gg C_{Y} \\
& C_{d} \gg C_{X} \\
& C_{Y}>C_{X} \\
& G_{Y d} \gg G_{X Y} \\
& P_{Z}=0
\end{aligned}
$$


sendo $\mathrm{K}_{\mathrm{S}}{ }^{\min }, \mathrm{R}_{\mathrm{XY}}{ }^{\min }, \mathrm{K}_{\mathrm{S}}{ }^{\max }$ e $\mathrm{R}_{\mathrm{XY}}{ }^{\max }$ os limites dos valores mínimo e máximo permitidos para o coeficiente Seebeck e a resistência elétrica. $\mathrm{O}$ valor de $\mathrm{P}_{\mathrm{Z}}$ deve ser mantido nulo para evitar que efeitos decorrentes de variações no mesmo sejam superpostos aos efeitos decorrentes de variações na corrente do TEM (Almeida, 2003).

Para quantificar a discrepância entre resultados experimentais e os dados do modelo real, é empregado o erro médio quadrático normalizado, dado por a função objetivo como descreve-se na Eq.(12).

$$
J_{M Q N}(\theta)=\sqrt{\frac{\sum_{i=1}^{N}\left(X_{i}-Y_{i}\right)^{2}}{\sum_{i=1}^{N} Y_{i}^{2}}} x 100 \%
$$

sendo que $\mathrm{X}_{\mathrm{i}}$ representa os dados do modelo real, $\mathrm{Y}_{\mathrm{i}}$ representa os dados experimentais, $\mathrm{N}$ é o número de pontos de dados e $\Theta=\left[C_{X} C_{Y} C_{d} G_{X Y} G_{Y d} G_{d a} K_{S} R_{X Y}\right]^{T}$ é o vetor de parâmetros a ser estimado

\section{VALIDAÇÃO DO MÉTODO}

As rotinas do método analisado no presente trabalho foram compiladas no software Matlab, no problema de estimação dos parâmetros contemplaram-se variações de população e fizeram-se mudanças ao número de iterações. Observou-se a efetividade do método atingindo erros menores de $1 \%$ na discrepância entre os resultados experimentais e o modelo empregado. Na Tab. 1 foi empregado o erro médio quadrático como medida para fazer a comparação entre os parâmetros estimados pelo método apresentado e os parâmetros do modelo real.

Tabela 1. Comparação dos parâmetros obtidos pelos métodos PSO e Modelo Real.

\begin{tabular}{c|c|c|c|c|c}
\hline Parâmetro & Max & Min & PSO & Modelo Real & \% Erro \\
\hline Cx (J.K-1) & 3 & 4 & 3,6 & 3,2 & 12,5 \\
\hline Cy (J.K-1) & 105 & 108 & 106 & 108 & 1,85 \\
\hline Cd (J.K-1) & 3000 & 4000 & 3790 & 3500 & 8,29 \\
\hline Ks (V.K-1) & 0,01 & 0,03 & 0,022 & 0,02 & 10 \\
\hline Rxy (Ohm) & 1 & 5 & 1,48 & 1,32 & 12,12 \\
\hline Gxy (W.K-1) & 0,1 & 0,5 & 0,26 & 0,23 & 11,11 \\
\hline Gda (W.K-1) & 10 & 20 & 13,8 & 10 & 38 \\
\hline Gyd (W.K-1) & 20 & 30 & 24,1 & 24 & 0,42 \\
\hline
\end{tabular}

Na Fig. 8 mostra-se que o comportamento do modelo estimado é aproximado quando comparado com o modelo real, mesmo quando o sinal de excitação apresenta mudanças súbitas no seu comportamento, pode-se notar que as discrepâncias entre os parâmetros obtidos por ambos os métodos, não significa incoerência, apenas que alguns dos parâmetros não geram grandes alterações no comportamento geral do sistema.

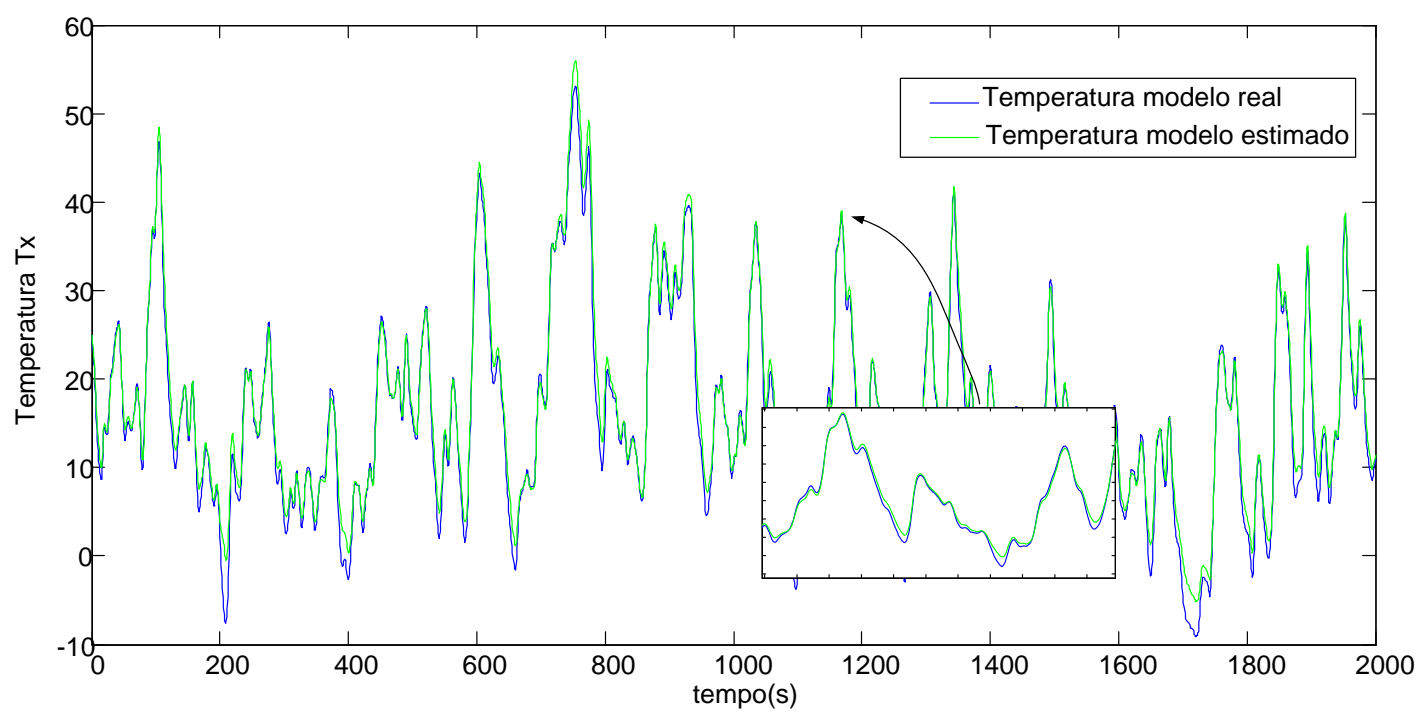

Figura 8. Temperatura da face superior do TEM obtida com o modelo real e o modelo PSO. 


\section{CONCLUSÃO}

No presente trabalho foi implementado um método estocástico para estimação dos oito parâmetros dinâmicos de um modelo termoelétrico TEM não linear, baseado na utilização do algoritmo PSO. O modelo do TEM foi inicialmente excitado com uma corrente do tipo ruído branco com componentes em alta e baixa frequência, assim excitar os modos dinâmicos do TEM que foram estimados. A corrente aplicada no TEM tem relação direta com a temperatura que é medida em uma das faces do TEM, os resultados das medições são fornecidos no algoritmo PSO, se tornando um trabalho complexo de otimização. O algoritmo do PSO estima os parâmetros que caracterizam o TEM tendo em consideração as restrições de valores mínimos e máximos de cada parâmetro do modelo. Os resultados obtidos para a estimação de parâmetros do TEM mediante o algoritmo PSO mostraram resultados com erros inferiores ao $1 \%$ quando comparados ao modelo real, comprovando assim a efetividade do modelo proposto. As discrepâncias entre os parâmetros obtidos por ambos os métodos, não significa incoerência, apenas que alguns dos parâmetros não geram grandes alterações no comportamento geral do sistema.

\section{AGRADECIMENTOS}

Os autores agradecem à CAPES, PROPG-UFABC e CNPq pelo apoio financeiro durante a pesquisa deste trabalho.

\section{REFERÊNCIAS}

Almeida, L. A. L. 2003. Modelo de Histerese para Transição Semicondutor-Metal em Filmes Finos de $\mathrm{VO}_{2}, \mathrm{PhD}$ thesis, Departamento de Engenharia Elétrica - UFPB, Campina Grande, Paraíba, Brasil.

Chávez, J. A., Ortega, J. A., Salazar, J., Turó, A. and García, M. J. 2000. Spice model of thermoelectric elements including thermal efects, Proceedings of the 17th IEEE Instrumentation and Measurement Technology Conference IMTC

Dalola, S., Ferrari, M., Ferrari, V., Guizzetti, M., Marioli, D. and Taroni, A. 2009. Characterization of Thermoelectric Modules for Powering Autonomous Sensors, IEEE Transactions on Instrumentation and Measurement, (Volume:58 ,Issue: 1 ).

Huang, B. J. and Duang, C. L. 2000. System dynamic model and temperature control of a thermoelectric cooler, International Journal of Refrigeration (23): 197-207.

Kennedy, J., Eberhart, R. 1955. Particle Swarm Optimization Washington, DC 20212, Purdue School of Engineering and Technology, Indianapolis IN 46202-5160.

Meijer, G. and Herwaarden, A. 1994. Thermal Sensors, IOP Publishing.

Mitrani, D., Tome, J., Salazar, J., Two, A., Garcia, M. and Chavez, J. 2004. Methodology for Extracting Thermoelectric Module Parameters. Sensor Systems Group, Electrical Engineering Department, Universitat Politecnica de Catalunya.

Mondal, D., Chakrabarti, A. and Sengupta, A. 2012. Optimal Placement and Parameter setting of SVC and TCSC using PSO to Mitigate Small Signal Stability Problem. Department of Electrical Engineering, Bengal Engineering and Science University, Howrah 711 103, India.

Ljung, L. 1987. System Identification: Theory for the User, Englewood Cliffs, NJ: Prentice-hall, 1987

Palacios, R., Arenas, A., Pecharromán, R. R. and Pagola, F. L. 2009. Analytical procedure to obtain internal parameters from performance curves of commercial thermoelectric modules. Universidad Pontificia Comillas, Instituto de Investigación Tecnológica, Alberto Aguilera, 23, 28015 Madrid, Spain.

Poli, R. 2007. Analysis of the Publications on the Applications of Particle Swarm Optimization. Department of Computer Science, University of Essex, Colchester CO4 3SQ, UK.

Rowe, D. M. 1995. CRC Handbook of Thermoelectrics, CRC Press.

Therdbankerd, T., Peerayot, S., Nattapon, Ch., and Hideaki, F., 2010. Parameter identification of a linear permanent magnet motor using particle swarm optimization, Department of Electrical Engineering, Kasetsart University 50 Phahonyothin Rd., Ladyao, Jatujak, Bangkok, 10903, Thailand

Vasconcelos, A. 2007. Algoritmos Genéticos e Particle Swarm Optimization e suas Aplicações Problemas de Guerra Eletrônica. Diretoria de Sistemas de Armas da Marinha, RJ. Brasil.

Venkata, R. and Vivek, P. 2012. Multi-objective optimization of two stage thermoelectric cooler using a modified teaching-learning-based optimization algorithm. Department of Mechanical Engineering, S.V. National Institute of Technology, Ichchanath, Surat 395 007, Gujarat, India.

\section{RESPONSABILIDADE AUTORAL}

"Os autores são os únicos responsáveis pelo conteúdo deste trabalho". 


\title{
THERMOELECTRIC PARAMETER ESTIMATION BASED ON METAHEURISTIC BEES
}

\author{
Daniel Ricardo Ojeda Giratá, daniel.ojeda@ufabc.edu.br ${ }^{1}$ \\ Omar Alexander Chura Vilcanqui, omarchu@gmail.com² \\ Luiz Alberto Luz de Almeida, luiz.almeida@ufabc.edu.br ${ }^{3}$ \\ ${ }^{1}$ Universidade Federal do ABC - Pós-Graduação em Engenharia Elétrica ... htttp://www.ufabc.edu.br/ \\ ${ }^{2}$ Universidade Federal da Bahía - Pós-Graduação em Engenharia Industrial ... http://www.ufba.br/ \\ ${ }^{3}$ Universidade Federal do ABC - Pós-Graduação em Engenharia Elétrica ... http://www.ufabc.edu.br/
}

\begin{abstract}
Thermoelectric Modules (TEM) are used in the power generation and construction of thermal cameras for material characterization such as smart memory allow (SMA), among other. To obtain a correct TEM representation, it is necessary a proper model identification procedure to represent the TEM operation, both in DC and other relevant frequencies. In this paper, a TEM model is proposed, where the nonlinear caracteristics were considered. Where classical methods for linear parameters estimation are not apply to the proposed model. To obtain the TEM parameters, it is excited by a current with a spectral power density of a white noise, and then is used the temperature response for the PSO algorithm (Particle Swarm optimization) to make the estimation. The chosen model is nonlinear with eight parameters, wich represent the TEM: the top layer, the hot side, the middle layer, cold side and the heatsink. For numerical stability, the white noise excitation is filtered before, geting a dynamically persistent signal with high and low frequencies components, so TEM will be properly characterized. Simulation results show the effectiveness of the PSO in TEM parameters estimation.
\end{abstract}

Keywords: thermoelectric module, parameters estimation, particle swarm optimization (PSO), methaeuristic optimization, nonlinear system identification. 